\title{
Creativity and Learning in Citizen Cyberscience - Lessons from the Citizen Cyberlab Summit
}

\author{
EGLE MARIJA RAMANAUSKAITE, Citizen Cyberlab \\ MORDECHAI HAKLAY, University College London
}

\begin{abstract}
This article summarizes the Citizen Cyberlab (CCL) Summit, which took place at University of Geneva on 17-18th September 2015, and introduces the special issue on "Learning and Creativity in Citizen Science". As the final event of a 3-year EU FP7 CCL project, the Summit sought to disseminate project results and reflect on the issue of citizen science (CS) as a participatory environment where opportunities for self-development and various types of creativity can arise. A number of interesting themes emerged at the intersection of the work presented by project collaborators and external partners, including the different types of creativity that are evident in $\mathrm{CS}$, the role of the community as the main medium for innovation and participant learning to occur, and the common challenges concerning the design, initiation and management of CS projects.

The current issue presents work done during the CCL project, as well as external project contributions, for which the main focus is on learning and creativity in CS. The set of articles addresses diverse aspects of the topic, ranging from empirical research on the phenomena themselves, to tools, platforms and frameworks developed specifically for citizen cyberscience (CCS) with creativity and learning in mind, and distinct CS cases where these phenomena manifest in previously undescribed and unexpected ways.

We hope that the issue will be useful to researchers and practitioners who aim to study, evaluate or design for learning and creativity in a range of CCS projects.
\end{abstract}

\section{INTRODUCTION}

Herein we present the findings reported at the Citizen Cyberlab (CCL) Summit, the culmination of a European project focused primarily on exploring learning and creativity in citizen cyberscience (CCS). The CCL Summit took place at the University of Geneva on 17-18th September 2015 as the final event of a 3-year EU FP7 CCL project. The event sought to disseminate project results and reflect on the issue of citizen science (CS) as a participatory environment, where opportunities for self-development and various types of creativity can arise. 
In this article we summarize the findings and considerations shared during the CCL summit that includes work from the project, as well as contributions of external project partners. We then introduce the diverse and informative compilation of articles that compose this special issue of Human Computation on Learning and Creativity in Citizen Science.

CS is becoming a popular way to engage the general public with science, whether it is through data collection, data analysis or both (Silvertown, 2009, Bonney et al., 2009), allowing even people with no scientific background to participate. Enabled by Web-based technology, a new way to do CS has emerged in recent years, which has been named citizen cyberscience (CCS) (Grey, 2009). CCS projects include a wide range of tech-aided activities, which according to Haklay (2013) can be subdivided into:

- volunteer computing (VC), which is based on installing particular software (e.g. $\mathrm{BOINC}^{1}$ ) and sharing individual computer resources to run complicated scientific algorithms (e.g. when the computer is idle). Example projects include SETI@home², Rosetta@home ${ }^{3}$ and many others.

- volunteer thinking (VT), which includes scientific data analysis, e.g. by classifying or annotating images, solving puzzles etc. Example projects include Galaxy Zoo ${ }^{4}$, Cell Slider ${ }^{5}$, Foldit $^{6}$ etc.

- participatory sensing (PS), which includes data collection, e.g. imaging biodiversity, sensing the environment and so on. Example projects include The Great Sunflower Project $^{7}$, NoiseTube ${ }^{8}$ and many others.

Considering the role of the Web and computer-based technology in most of our daily lives, the rising popularity of CCS is not unexpected. Citizen scientists can now connect to Web-based projects wherever they are and whenever they have time. For example, one can log into a cancer research game ${ }^{9}$ through an app on their phone, contribute to humanitarian aid ${ }^{10}$ in short bursts by classifying photos during working breaks, etc.

Citizen scientists usually join such CS initiatives prompted by curiosity, desire to contribute to science and have a fun time (Curtis, 2015; Iacovides et al., 2013; Rotman et al., 2012). However, educational potential, including learning more about the subject and understanding of the scientific process, are often proposed as possible "side effects" in CS (Bonney et al., 2009;

\footnotetext{
${ }^{1}$ https://boinc.berkeley.edu/

2 http://setiathome.ssl.berkeley.edu/

3 https://boinc.bakerlab.org/

${ }^{4}$ http://www.galaxyzoo.org/

5 http://www.cellslider.net/\#/

6 https://fold.it/portal/

7 http://www.greatsunflower.org/

8 http://noisetube.net/\#\&panel1-1

9 http://www.cancerresearchuk.org/support-us/play-to-cure-genes-in-space

${ }^{10}$ For example, GeoTag-X - http://geotagx.org/
} 
Cronje, 2011; Silvertown, 2009; and others). Though it is not too difficult to imagine that such informal learning ${ }^{11}$ could take place in participatory scientific activities (Bell et al., 2009), considering the highly individual, "patchy" and sometimes incidental nature of informal learning (Marsick \& Watkins, 2001), measuring what and how citizen scientists learn has remained a challenge (Cronje et al., 2011).

In addition to learning itself, CCS has been considered a potential ground for creativity, as participants are free to experiment, develop new ideas and problem-solving techniques and "think outside the box", especially where scientists fail to do so (Dickinson, 2011). Citizen scientists have been known to make important scientific discoveries, for example, the deciphering of an HIV-related protein structure that perplexed scientists for decades (Khatib et al., 2011), and the discovery of a new type of galaxy (Cardamone et al., 2009), the latter being subject to ground breaking astrophysics research ever since ${ }^{12}$. Of course, more everyday-type creativity could also take place in CCS, such as discussing problem solutions in forums or artistic expression based on activities within the projects. However, as there is neither a common consensus of what creativity is (Batey, 2012), nor a framework for such an analysis in the context of CCS, the evidence has remained patchy and limited to individual case reports.

The 3-year CCL project aimed to fill the existing gap regarding understanding of what and how learning and creativity manifests in CS, focusing mainly on projects enabled by the Internet technology (CCS).

\section{CITIZEN CYBERLAB PROJECT}

The CCL project, carried under EU Seventh Framework Programme, set out in 2012 to tackle the popular, yet poorly researched issues of learning and creativity in online collaborative spaces, and both to provide the much needed fundamental research, and develop new platforms, tools and pilot projects, that would specifically aim to foster learning and creativity in CCS.

The project was carried out by seven international partners, including the European Organization for Nuclear Research (CERN), United Nations Institute for Training and Research (UNITAR/UNOSAT), Imperial College (ICSTM), UCL Interaction Centre (UCLIC) and Extreme Citizen Science (ExCiteS) at University College London (UCL), Université de Genève (UNIGE), Université Paris Descartes (UPD), and a UK-based innovation consultancy, The Mobile Collective (TMC). CCL partners set out to develop:

1. Tools: IdeaWeave, an online "creativity incubator" for collaborative development of ideas and projects; EpiCollect+, a generic data collection app, which allows easy gathering of multimedia-rich and geotagged data; and CCL Tracker, which uses web analytics to track participant behaviour and learning in Web-based platforms.

\footnotetext{
${ }^{11}$ Informal learning, which includes incidental learning, is not typically classroom-based or highly Structured; control of learning rests primarily in the hands of the learner, and it takes place "wherever people have the need, motivation, and opportunity for learning" (Marsick \& Watkins, 2001).

${ }^{12}$ http://blog.galaxyzoo.org/?s=green+peas
} 
2. Platforms: CitizenGrid, which allows easy setup of VC and VT projects; RedWire, which is an open platform for creating and "mashing up" games, including CS and educational games, and RedMetrics which is used to track game analytics; and GeoKey which enables collaborative mapping and problem solving efforts mainly in the contexts of participatory research.

3. Pilots, which are composed of different Tool/Platform combinations, and aim to "test" the potential for learning and creativity in such contexts: Virtual Atom Smasher (VAS), a particle physics game; GeoTag-X, which asks to annotate and geo-tag images in disaster relief settings; Synthetic Biology, which consists of different projects to involve the general public in synthetic biology research; and Extreme Citizen Science, which concentrates on grass-roots participatory sensing projects, set up by citizen scientists themselves.

The overall project architecture and the links between pilots, platforms and tools, is summarized in Figure 1.
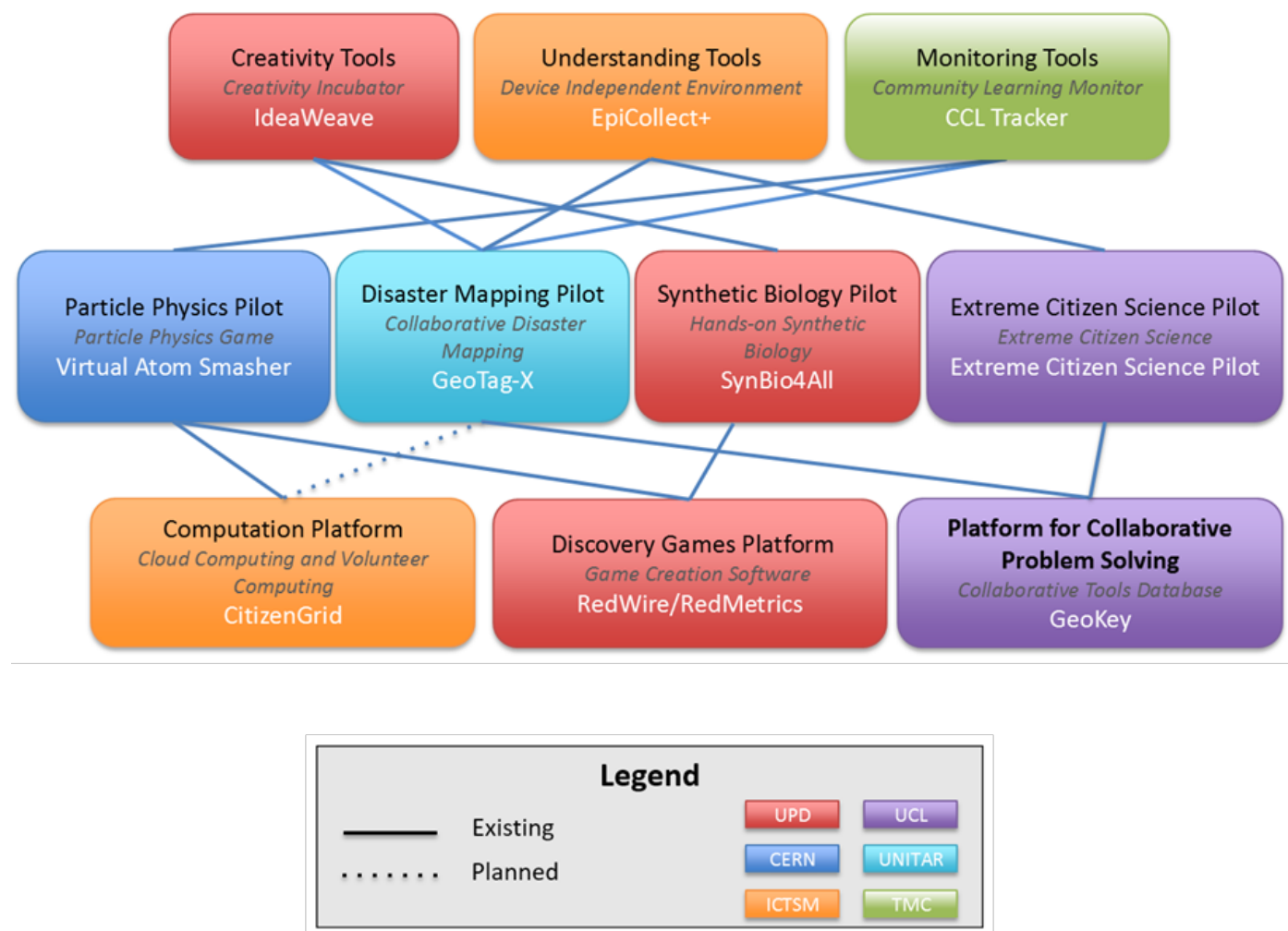

Figure 1. Components of Citizen Cyberlab. Tools, platforms and pilots are colour-coded to represent CCL partners who were leading the development of each. Lines correspond to 


\section{complete and planned links between the different components of the project, i.e. tools and platforms that have been integrated into relevant pilots.}

\section{LESSONS FROM THE CITIZEN CYBERLAB SUMMIT}

The Citizen Cyberlab Summit took place on 17-18th September and was the final event carried out by the full CCL consortium. It was organized as a focused and intimate gathering, to allow project partners to disseminate outcomes and gain support for the final stages. The summit concentrated on the CCL activities, and aimed to share the project findings with an international group of academics, practitioners and community members, who are involved in CCS, and learning and creativity research. The event combined a series of seminar-style talks and demos, with breakout sessions designed for intense discussions and brainstorming to take place, thus following the traditional "unconference" style of ThinkCamps which have been organized throughout the course of the project. The summit gathered 40 participants from around the world. The event was structured around four sessions which allowed detailed discussion of the project results and plans for development beyond the project end.

The Citizen Cyberlab Summit was dedicated to enhancing the opportunities for learning and creativity in CCS. Through short talks, demo sessions and group discussions, the objective of the first day of this summit was to explore how software platforms, tools and pilot projects can be designed to improve learning and creativity opportunities in CCS. The second day explored how quantitative and qualitative methods may be used to evaluate the learning experience and creative potential of CCS, as well as addressing the issue of sustainability of projects and communities in CCS.

\subsection{CCS is a medium for different kinds of creativity}

As one of the core topics of the project, creativity was discussed extensively during the Citizen Cyberlab Summit, with CCL research findings presented by Dr. Charlene Jennett (UCLIC) and brainstorming discussions that followed.

Considering the fact that creativity is a much debated concept as it is, and no real consensus on what does and does not count as "creative" exists, defining creativity in CCS is no easy task. However, the research conducted within the project, as well as examples shared by CCL pilot owners and external collaborators, revealed ample ways of how both "special" and "everyday" creativity happens.

During the first year of the project, UCLIC and UNIGE conducted interviews with a wide range of existing CS project participants, including the BOINC community, EyeWire ${ }^{13}$, Transcribe Bentham $^{14}$, Bat Detective ${ }^{15}$, Zooniverse ${ }^{16}$ and Mapping for Change ${ }^{17}$ projects. The idea was to

\footnotetext{
${ }^{13}$ http://eyewire.org/explore

14 http://blogs.ucl.ac.uk/transcribe-bentham/

15 http://www.batdetective.org/

16 https://www.zooniverse.org/
} 
grasp the scope of creativity in different projects, powered by different technologies and contexts. A number of examples of creativity emerged, including the already mentioned "Green Peas", the discovery of which owes it to the participants" own motivation and "Give Green Peas a chance" campaign; creation of chatbots, which can answer FAQs in a community forum by EyeWire participants; drawing pictures of historic ships that were being transcribed in Old Weather project; and others (Jennett et al., 2016a).
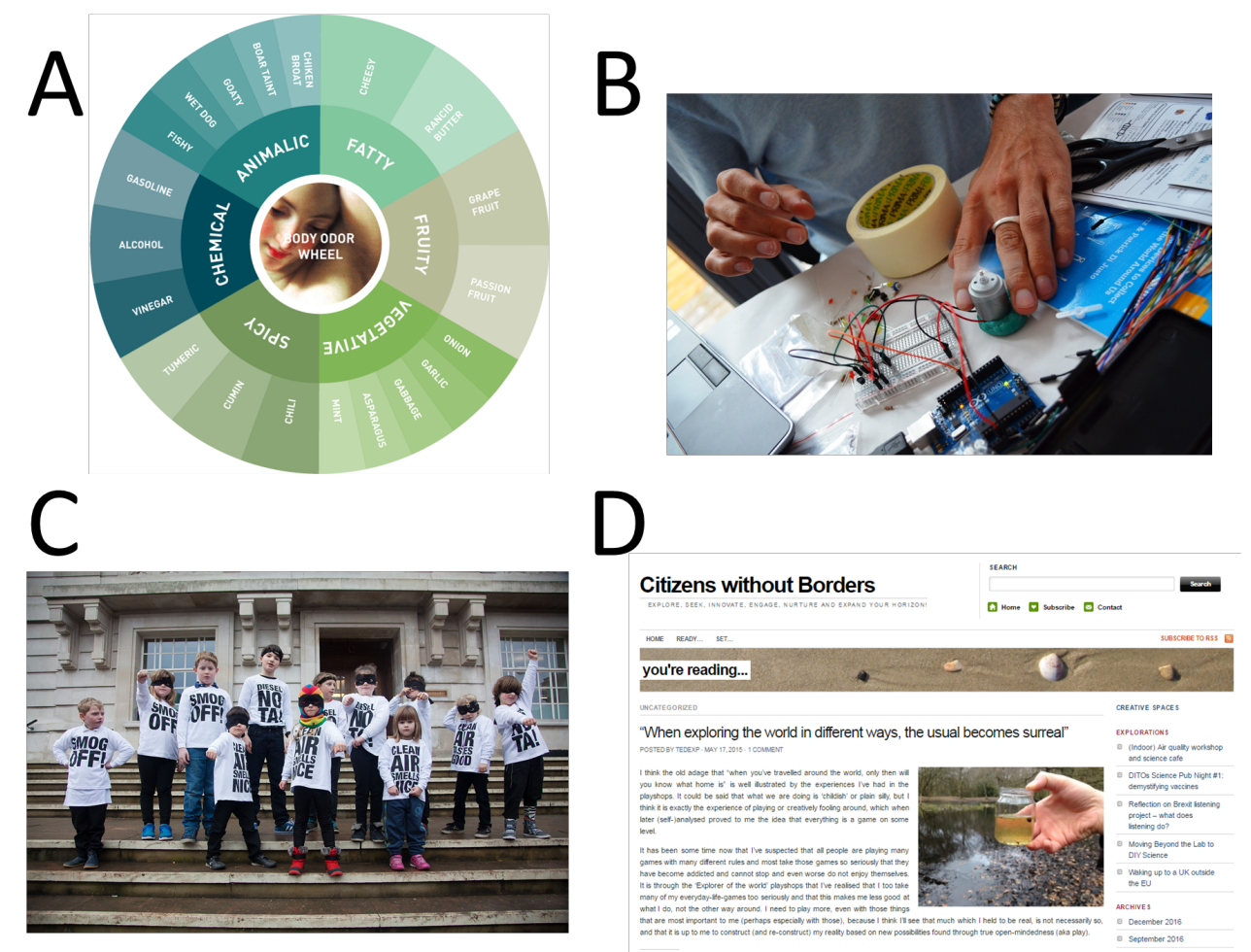

Figure 2. Examples of creativity in CCL pilot projects. A - "The Smell Game" designed by Synthetic Biology students to analyzing body odor samples from the public (C) 2014.iGem.org); B - DIY creative tools in Extreme Citizen Science projects (Cindy Regalado, Bere Architects, and the Mildmay Community Centre); C - "I like clean air" T-shirt campaign (C) I LIKE CLEAN AIR); D - blog post by a "Citizens without Borders" participant (https://citizenswithoutbordersdotcom.wordpress.com/).

Creativity within the Citizen Cyberlab was explored by interviewing participants and project owners from the four pilots: GeoTag-X, Synthetic Biology, VAS and Extreme Citizen Science. The results are reported in full in Jennett et al. (2016b) within this issue. However, below are several examples of creativity that were observed:

${ }^{17}$ http://mappingforchange.org.uk/ 
- GeoTag-X volunteer-developers, who help to design new projects, see this process as the most creative; however volunteer-analysers, who only join in to classify and annotate images see some of the more complicated tasks, including the geo-referencing of pictures (e.g. marking the geographical location of a cultural site) to also be creative, especially when geo-tagging techniques can be shared with other participants to find creative solutions. The latter observation is particularly curious, since, as explained by GeoTag-X project owner, geo-tagging has been perceived as the hardest task by the project participants, as revealed by participant surveys.

- In the Synthetic Biology pilot, students have been creating games and videos (Figure 2, A), which are meant to promote the synthetic biology projects to the wider public.

- Creativity within the VAS has been mostly linked to idea sharing and collaborative learning.

- In Extreme Citizen Science, the DIY nature of tools and projects is in itself an important part of creativity (Figure 2, B); people also show their work and express their opinions in blogs (Figure 2, D).

- Other examples included volunteers creating T-shirts to promote the project within their community (Figure 2, C); participants creating better visuals, e.g. maps, if they are not happy with the ones available in the project.

The CCL external partners and advisors also shared the following examples of creativity, as observed in their own projects:

- in Crowdcrafting.org ${ }^{18}$, when people are not active for a month, they get an email telling them that they will be deleted; in response to this, one participant created a small application that generates tweets from WWI transcriptions on the day exactly 100 years after it happened, thus generating regular activity that is connected to the project;

- in Cornell Lab of Ornithology, volunteers suggest new protocols and tasks, i.e. new ways to improve the projects;

- the games of Science@Home are targeted specifically to explore when problem solving becomes creative - this happens mainly by using the tools creatively and explaining to the researchers how they solve issues;

- in World Community Grid (WCG) $)^{19}$ one volunteer created graphics from the API that other volunteers now use and expect to see it as part of the project.

Clearly, some of these examples more related to scientific discovery (the "Eureka moment" type of creativity) than others, and some are not linked to direct project scientific outcomes at all. Yet one could argue that imaginative self-expression (such as artwork) and solving project problems (such as creating chatbots or suggesting project improvements) have value on their own: whether on individual, community or project level.

\footnotetext{
${ }^{18}$ http://crowdcrafting.org/

19 https://www.worldcommunitygrid.org/
} 


\subsection{Keeping lines of communication open is important}

One of the most important findings of the research was that people seem to get creative when they are driven by a desire to share something with the community (also see Jennett et al. (2013). As suggested by the teams at UCLIC, UNIGE and TMC, creating a communication-friendly space within the project should therefore be one of the top priorities for new CS projects. Specific tips for the projects include: providing communication channels via forum and/or social media, organizing community events (e.g. BOINC community organizes computing competitions), and role management - i.e. if you can see someone is doing well, encourage them to take more responsibility.

A particularly important point made was also keeping lines of communication open between the scientists and the participants, including providing progress feedback both on individual and project level (also see Jennett \& Cox (2014). These findings were similar to those of other researchers. For example, Tina Phillips from Cornell Lab of Ornithology, who also presented her research on learning in CS during the summit, highlighted the tendency of lower engagers to feel less connected to CS projects, and crave more social outlets in response.

Interestingly, a number of these considerations were shared by external CCL partners, who have observed similar dynamics in their own projects. For example, Juan Hindo from WCG, a volunteer computing (VC) platform that was presented in the summit, also highlighted the "storytelling about the science behind", i.e. letting the volunteers know exactly what research they are contributing to and how, as one of their engagement strategies. One of the most effective strategies to improve volunteer engagement in WCG has in fact been consistent and regular updates on the research, articulating the value of volunteer contributions by telling research success stories etc. A good idea, in WCG's experience, is also to give volunteers control over communication they would like to receive, in such a way providing them with "a voice".

\subsection{Design of "the box" - no one-size-fits-all}

How do we design "the box" so people can get out of it? - Laure Kloetzer, Citizen Cyberlab Summit

The design question was subject to extensive discussion within the summit, with most opinions falling into one of the two groups: 1) creativity should have completely free reign, i.e. enough possibilities created for users, and 2) creativity should be structured, i.e. participants allowed to operate only in limited conditions. For example, according to Jacob Sherson (University of Aarhus), their Science@ home $^{20}$ games only allow players to operate in specific modes, which then encourages them to find the best combinations and problem solutions with options available to them.

Another problem is the need to accommodate the myriad of volunteers whose participation patterns differ significantly. For example, some participants would want to be fairly passive and

${ }^{20}$ https://www.scienceathome.org/ 
have low involvement, while others might want to do much more. An important point in this respect was made by Michael Weber, who leads Rechenkraft.net ${ }^{21}$ VC community. According to him, volunteers can only do so much within CCS environment alone. For example, the main force behind Rechenkraft.net, which not only gathers a community of active VC enthusiasts, but also hosts its own VC project - RNA World ${ }^{22}$, was an opportunity to create a council-supported local hackspace. Therefore, the volunteers, the infrastructure and the support together form the "magic" combination that allows creativity to happen, and should be considered in the design of all CS projects.

Though discussions about designing for creativity seemed to have opened more questions than they answered, what we see here is the beginnings of an active thought process that should accompany creation of any CS project in the future, as the creative and innovative potential of such projects is becoming obvious.

Some guidelines to the design of CS projects in regards to enhancing the opportunities for learning and creativity, are discussed in Yadav \& Darlington (2016a) within this issue.

\subsection{Learning happens almost universally in CS, but is project and user specific}

Research lead by the team at UNIGE looked at what learning and how happens in the context of CCS. Results that emerged from initial interviews with existing CCS project participants during the first year of the CCL project allowed the description of 3 levels of learning including: 1) the project, e.g. science behind; 2) the task, e.g. task mechanics; and 3) community, e.g. peer-to-peer learning (Kloetzer et al., 2013). However, this was not enough to understand what and how people learn in great detail, and the research that followed was focused on just this issue.

The question of how people learn was explored by the team with a large scale ILICS (Informal Learning in Citizen Science) survey conducted during the third year of the project, on the basis of the previously defined levels and types of learning that occurs in CS. About 700 respondents from a range of CS projects, including VC, VT and other types of CS, participated in the survey. Strikingly, the results revealed that most $(86 \%)$ of the participants in CS projects learn at least something, even though learning might not be their primary motivation, nor one of the explicit goals of project creators. It is important to note, however, that learning seems to be very diverse and multidimensional, and cannot be generalized for all volunteers. On the other hand, valuable patterns emerge from research already collected, e.g. groups of specific types of learners in CS, which could inform the design of such projects significantly.

For example, ILICS survey results revealed the following typology of learners:

- Type $1(6 \%)$ learns by contributing and using external resources;

- $\quad$ Type $2(11 \%)$ by discussion and other means;

- Type $3(13 \%)$ learns through practicing and some external documentation;

\footnotetext{
${ }^{21}$ http://www.rechenkraft.net//index_en.html

22 http://www.rnaworld.de/rnaworld/
} 
- $\quad$ Type 4 (4\%) use other (not identified) means;

- $\quad$ Type $5(15 \%)$ learn through practicing and reading project documentation;

- Type 6 participants (30\%) learn mostly through project documentation.

Interestingly, cross-linking the types of learning process ("how") to the feeling of learning, social learners seem to report they learn more than the other types of learners. These findings, according to the researchers, has provided evidence to the main working hypotheses of the CCL learning research, including the prediction that people learn in different ways, and that empowerment of the participants, including the creation of a community and creation of roles, leads to richer learning outcomes.

In terms of the "what" people learn question, most respondents $(88,5 \%)$ felt they learned on-topic knowledge (about the domain itself) at least to an extent, scientific skills $(79,9 \%)$, technological skills $(61,9 \%)$, technical skills $(58,4 \%)$, political skills $(52,1 \%)$, collaboration skills $(53 \%)$ and communication skills $(53,8 \%)$ and organizational skills $(55,3 \%)$.

Importantly, research findings on learning in CS gathered by UNIGE resonated well with those of Cornell Lab of Ornithology, presented by T. Phillips. Their research looked at contributory, collaborative and co-created projects - including NestWatch ${ }^{23}, \mathrm{CoCoRaHS}^{24}$, and Global Community Monitor ${ }^{25}$. They had 83 interviews from low, medium and high contributors and information from project leaders. Preliminary data analysis revealed that data collection, communicating with others and understanding protocols are very common participant activities in CS.

The research also demonstrated that while all volunteers collect data in the studied projects, a proportion also feel part of the community, and group projects might have more impact than individual ones.

\subsection{Engagement and identity foster learning and creativity}

Participants are not just learning - they are talking about leaving a legacy behind! - Tina Phillips (Cornell Lab of Ornithology), Citizen Cyberlab Summit

The issue of participant engagement through "roles" and building an "identity" resurfaced on multiple occasions during the summit. Findings gathered through CCL ILICS study revealed that regarding social engagement, about $11 \%$ of participants take part in the community, and for $61 \%$ it was the first time in their life that they took such a role. Importantly, such roles seem to be diverse in nature, e.g. ranging from forum management to creation of new data visualization tools. This highlights the importance of CS as a medium for new types of self-expression and engagement.

\footnotetext{
${ }^{23}$ http://nestwatch.org/

24 http://www.cocorahs.org/

25 http://www.gcmonitor.org/
} 
Importantly, both the CCL creativity and learning research teams (UCLIC and UNIGE) and T. Phillips from Cornell Lab of Ornithology have proposed independent models, which in one way or another link participant engagement and identity with learning and creativity. The "MLC model" was proposed by CCL researchers after the exploratory CCS interviews during the first year of the project (Jennett, 2016a), suggesting multiple feedback loops, including a positive link between engagement and learning, which leads to creativity (Figure 3). For example, initial motivation to participate allows volunteers to gain new skills, which in turn lead to increased selfconfidence and ability to share their knowledge and skills with the community, and potentially gain a different status, e.g. as a project moderator. Such new roles may also lead to self-reflection and awareness of one's own learning. In such a way, community supports the development of confidence and identity as a science learner. Increasing engagement and learning may also lead to higher potential and confidence for self-expression, creating ample opportunities for creativity to take place.

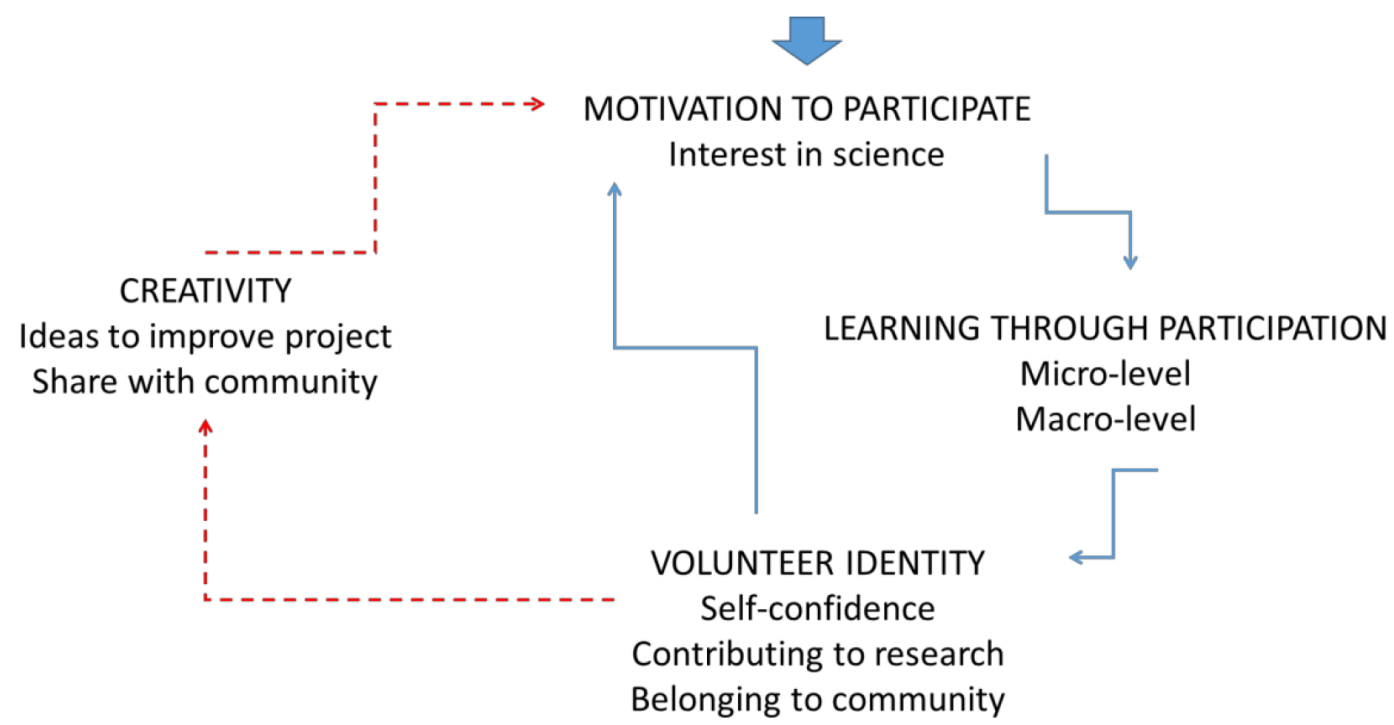

Figure 3. According to Jennett et al. (2016a) a positive feedback loop exists between
motivation, learning, building an identity and creativity within CCS.

A similar working model was also described by T. Phillips from Cornell Lab of Ornithology, who observed links between engagement, learning and identity in CS projects. According to their research, majority of volunteers join CS projects with a high interest in science and/or nature as well as concern over some environmental issue, and most are immediately aware of the concept of CS and their own role in it. They are examining whether deeper engagement results in more learning outcomes, which also seem to be related to scientific identity taken up by the volunteers. Fascinatingly, the most engaged volunteers seem to be keen to learn primarily so they could teach others, or are even speaking about making a long term impact on the community of learners. For 
example, one of the respondents from NestWatch project reported that "training other people is one way to pass on skills that can go further than just me looking in boxes and enjoying the birds for myself."

Similar results were also obtained during CCL exploratory interviews early in the project, suggesting volunteers want to do things right, not just for themselves, but because they feel their work is valuable to a community of peers (Jennett et al., 2016a). As such, both research conducted within the CCL and the Cornell Lab of Ornithology suggest the most intense learning experiences are created with the community, and group projects might have more collective impact than individual ones, but more research is needed.

\subsection{CS is subject to the $1 \%$ rule}

Despite the evidence of learning and creativity occurring across different CS projects, one of the most pressing issues for CS project owners and researchers is understanding why a divide between "low" and "high" contributors happens. For example, CCL research revealed on multiple occasions that in addition to the fact that out of the whole population, only a small group of people participates in CS, only a tiny group of those participants do most of the work within the project (the $1 \%$ rule of the Internet culture ${ }^{26}$ ). For example, in Transcribe Bentham, a modest group of 16 volunteers have completed most of the tasks (Causer \& Terras, 2014).

Similar dynamics were observed by most participants at the Citizen Cyberlab Summit. For example, GeoTag-X, which was presented by Eleanor Rusack (UNITAR), has also seen a small group of "dedicated" contributors, while others seem to contribute in short bursts. Other CCL and external contributors have also highlighted similar experiences.

Of particular interest in this respect has been the recent research of WCG, which aimed to understand volunteer engagement and improve it as much as possible. Extensive volunteer study has revealed that most VC participants are men, 20-40 years old, and join with the primary incentive to help science. Importantly, learning about science was found to be an important reason to stay. According to J. Hindo, people want to understand the impact of the computations that they perform and this information has to be understandable (i.e. beyond plain statistics).

Based on these results, WCG team is now trying to build a more diverse volunteer base, provide more approachable scientific content and better articulate the value of volunteer contribution. Importantly, WCG team sees an opportunity to reach out to underrepresented populations. Their main engagement strategy is through the story-telling about the science behind, and ensuring people that the process is safe.

Understanding the demographics of CCS participants, and its possible relation to participant behaviour and learning has also been highlighted by Jose Luis Fernandez-Marquez (UNIGE), who has been developing the CCL Tracker framework. The framework uses Google analytics to gain deeper understanding of participant behaviour within CCS web platforms with an insight

${ }^{26}$ https://en.wikipedia.org/wiki/1\%25_rule_(Internet_culture) 
into their demographic profiles provided by Google. Further research could possibly bring insights into why only a small amount of volunteers has the lion's share within projects, and how the volunteer basis could be further enlarged. CCL Tracker is reported in Fernandez-Marquez et al. (2016) within this issue.

\subsection{Lowering barriers to participation}

One of the points that are almost universally agreed upon, is the fact that citizen scientists usually join CS projects due to a high interest in science, and are engaged in a wide range of activities even beyond the project itself. They often have an awareness of "contributing to something bigger", and crave that their work is used somewhere.

The latter point has been highlighted exceptionally by the research of UCLIC and UNIGE, Cornell Lab of Ornithology and WCG. In particular, the researchers report an almost universal requirement of ongoing feedback for project volunteers. For example, research conducted by the Cornell Lab of Ornithology revealed that people are concerned about how the data is used, and what is done with it, even if they will not analyze it themselves. This is, for example, accommodated by, providing raw data to the participants in $\mathrm{eBird}^{27}$ project. Similarly in WCG, volunteers are particularly motivated by the open data policy, which assures them that no one company or organization will hold ownership over the data they helped to collect. Naturally, these observations highlight the importance of transparency on all project levels, and are particularly relevant to the question of "moral economies". The latter, according to Bruno Strasser, who presented the History of the Citizen Sciences at the summit, is one of the fundamental elements that may motivate or keep citizen scientists from contributing, i.e. the question of why and what are volunteers doing it for: science, people, team, themselves, fun, or something else?

Another important consideration in terms of lowering barriers to CCS, is enabling people across different literacy levels and demographic variables to contribute. Of particular importance here is the observation that was made from ILICS study, suggesting that VC projects allow less educated volunteers to join, yet educational potential of such projects seems to differ little from the other types of CS (see also Kloetzer, et al. (2016) within this issue).

Extreme Citizen Science ${ }^{28}$ - one of the pilots of the CCL, also serves as an important example in this case. According to Muki Haklay, who presented Extreme Citizen Science at the summit, the approach has a potential to involve participants across different levels of literacy, as the extrinsic motivations of volunteers (e.g. to tackle local environmental issues), and the simple nature of DIY tools that are used in such projects allow a diverse range of people to join. Thus opportunities of learning and creativity are opened up for the part of the public who may not have access to them otherwise.

\footnotetext{
${ }^{27}$ http://ebird.org/content/ebird/

${ }^{28}$ Extreme Citizen Science is defined as a "situated, bottom-up practice that takes into account local needs, practices and culture" (https://www.ucl.ac.uk/excites).
} 
Making it easier to create community-driven, co-created or contributory CCS projects is also important to enable and improve participation. Platforms and tools presented during the Citizen Cyberlab Summit have been developed just for this purpose. For example, Citizen Grid, developed as part of the CCL project is a CCS platform dedicated for easy hosting of VC and VT projects. Crowdcrafting.org ${ }^{29}$, which has been developed in collaboration with the Citizen Cyberlab $^{30}$, allows CCS project creation (based on, e.g. image, video, audio analysis and annotation etc.) with a simple walk-through wizard, ensuring no programming knowledge is necessary, which could greatly improve the chances for more volunteer-led projects to emerge. One of the CCL project tools, EpiCollect+ serves a generic data collection tool that can be used on any smart device, and set up by anyone. RedWire is a CCL game platform, which allows mash-ups of existing games for easy creation of CS or educational games.

Another great example is the GeoTag-X pilot, which allows volunteers to contribute without any prior knowledge - content-related or otherwise, greatly lowering the barriers to joining. The participants are also walked through each project with specific tutorials, and supported by the community of scientists and other volunteers if issues arise. An important point that was put forward during GeoTag-X brainstorming discussions in the summit was "expectation management" - letting the participants know how are they expected to contribute, do they need to have a certain level of expertise (which is usually not the case in typical CCS project, meaning volunteers should be assured of that), how complicated is a task by nature and how long is it likely to take etc. Knowing what to expect, and what is expected of them, might help CCS participants to gain confidence quicker within the projects.

\section{FINAL REMARKS}

This overview of the Citizen Cyberlab Summit, which was organized with a primary aim to share and disseminate the final results of the 3-year CCL project, highlights the key take-home messages from the presented project results, and the important discussions that emerged with external CCL partners, who on many occasions shared similar experiences and considerations.

One of the greatest impacts of the CCL project, has been the ability to explore learning and creativity as it takes place in diverse types of CS projects, each powered by different contexts and different technologies. This level of diversity has been absent in the previous literature, which has been sporadic and largely limited to individual projects (e.g., Bonney et al., (2009); Brossard et al. (2005); Crall et al. (2013); Cronje et al. (2011); Jordan et al. (2011); Price \& Lee (2013)).

Due to the incidental, unstructured and even unexpected nature of informal learning, it is particularly difficult to pin-point exactly what and how participants are learning, especially if diverse learning environments are under study. However, triangulation by exploring both quantitative and qualitative data of learning, in the scope of CCS projects as well as CCL pilots,

\footnotetext{
${ }^{29}$ http://crowdcrafting.org/

${ }^{30}$ http://citizencyberlab.org/, originally called Citizen Cyberscience Centre.
} 
has allowed CCL project collaborators to unveil a range of interesting learning processes and outcomes. One of the most important considerations has been the community-driven learning that takes place in a variety of forms. Even considering the preliminary nature of some of the research findings, these observations agree with those of other researchers, and have theoretical ground in sociocultural learning (Vygotsky, 1978), situated learning and community of practice theories (Lave \& Wenger, 1991) among others, and were reported as consistent with observations of other researchers and practitioners who shared their input during the summit.

Even though the "1\% rule" reflects the participant distribution in most CCS projects today, since only a set of volunteers are highly engaged, these projects become real opportunity spaces for the active core of participants. Here engaged participants can gain new skills and experiences and meet new people with similar interests, all of which they could not have achieved easily in their everyday environments. The same applies for creative opportunities, which take various shapes within different CCS projects. Even though the creativity is perceived differently by all, including researchers, project creators and participants themselves, there is no doubt CCS serves as a rich medium for both "everyday" and "special" types of creativity to take place, especially when combined with high participant engagement and identity development.

One of the most important considerations for project creators is therefore a community-friendly design of projects, in which lines of communication are open both between different participants and between the scientists and the volunteers. Even if no one-size-fits-all answer exists, CS environments should foster the participants' desire to contribute and understand their contribution to science, and create a safe environment to explore, learn and create.

\section{STRUCTURE OF THE SPECIAL ISSUE}

The "Learning and Creativity in Citizen Science" special issue expands on the work presented at the CCL Summit, presents the main lessons learned during the 3-year CCL project, and incorporates rich contributions from CCL collaborators in Europe and worldwide.

The first paper of the issue taps into a previously unexplored dimension of CCS: volunteer computing. Even though participation in such projects is usually seen as passive, since the "science" is conducted by the volunteers' personal computers (PCs) during their idle time, authors reveal that a proportion of participants are actively engaged in community-driven learning. This happens mainly through community-led gamification, and leads to a range of learning outcomes, including improving one's computer and Internet literacy, scientific knowledge and literacy, communication and social skills. The research is presented in "Not so passive: engagement and learning in Volunteer Computing projects" by Kloetzer et al. (2016).

Expanding on the topic of measuring learning is "Data Analytics in Citizen Cyberscience: Evaluating Participant Learning and Engagement with Analytics" by Abu Amsha et al. (2016). Based on examples of different types of CCS: volunteer thinking and volunteer gaming, the authors demonstrate the best practices regarding the use of learning analytics, which could be used to evaluate learning and engagement in online CS projects. 
One example of a learning analytics package - CCLTracker, one of the tools developed during the CCL project, is described in detail in "CCLTracker Framework: Monitoring user learning and activity in web based citizen science projects" by Fernandez-Marquez et al. (2016). The authors present the CCLTracker analytics framework that can be easily linked to other online analytics tools, e.g. Google Analytics or Piwik, overcoming their integral limitations and improving data gathering concerning participant and potential learning behavior online.

RedMetrics, an open source game analytics solution, is presented next in "Improving Citizen Science Games through Open Analytics Data" by Himmelstein et al. (2016). RedMetrics can gather data from any platform (web, PC, console, etc.) and store it on an open repository. According to the authors, the use of such metrics can not only help improve scientific and CS games, but allow analysis of gamers' progress that could be useful for game researchers and teachers using games as a learning tool. Developed during the CCL project, RedMetrics serves as a specific instance of analytics used in online CS.

Next among the platforms created with learning and creativity in mind is GeoKey - an open source mapping tool that supports participatory mapping, often happening at a local community level. In "GeoKey - open infrastructure for community mapping and science" Roick, Haklay, \& Ellul (2016) present the architecture of GeoKey, a platform created as part of the CCL project, both as a data capture and data sharing tool, and describe its design elements that support learning and creativity in such projects.

“The smell of us - crowdsourcing human body odor evaluation" by Benony et al. (2016) reports results of a crowdsourcing application to evaluate human body odor. With the help of a webbased graphical interface that is informative, interesting, and fun, the researchers were able to demonstrate the value of an alternative method to analyze human body samples, all the while allowing the participants to learn about their own body odor and that of others in a fun and engaging way. The project was set up as part of the CCL Synthetic Biology pilot.

Volunteers' experiences of creativity in CCS, including the four CCL pilot projects: GeoTag-X, Virtual Atom Smasher, Synthetic Biology and Extreme Citizen Science, is explored in an interview-based study "Creativity in Online Citizen Science: Interviews with Volunteers of the Citizen Cyberlab Pilot Projects" by (Jennett et al., 2016b). By using an interview approach the authors were able to reveal a range of creative activities that happen in CCS projects, including suggesting project improvements, producing artwork, creative writing, taking up outreach activities, and even the development of new research projects. As seems to be the case with learning, creativity seems to be fostered by a supportive environment and a sense of community in $\mathrm{CCS}$.

Design considerations that address the dynamic link between participation, learning and creativity are explored in "Design Guidelines for the User-Centered Collaborative Citizen Science Platforms" by Yadav \& Darlington (2016a). The presented guidelines are illustrated with casestudies on existing CS platforms, including CitizenGrid, developed part of the CCL project, as well as Zooniverse, World Community Grid, CrowdCrafting and EpiCollect + . 
For the benefit of new CS project creators, "Conceptual Frameworks for Building Online Citizen Science Projects" Yadav \& Darlington (2016b) present a series of conceptual frameworks meant to guide in every step of creating a new CS project. The frameworks are based on existing CS projects, and include categorization, decision and deployment frameworks, which take all types of CCS, including often unrepresented volunteer computing, into account.

Lastly, the issue is concluded with three articles demonstrating the power of human computation and the learning opportunities creating by specific CS projects: "Agroecology: A Fertile Field for Human Computation" by Hanappe et al. (2016), "A Crowdsourcing-based Air Pollution Measurement System Using DIY Atomic Force Microscopes” by Lopez Martinez et al. (2016), and "Landfill Hunter: Learning about Waste through Public Participation" by Johnson (2016).

We hope that the issue will be useful to researchers and practitioners who aim to study, evaluate or design for learning and creativity in a range of CCS projects.

\section{AKNOWLEDGEMENTS}

We would like to thank all of the CCL summit participants for sharing their work during the event, and to Institut Confucius de l'Université de Genève for kindly hosting the summit. We are grateful to Charlene Jennett, Oula Abu Amsha and Tina Phillips for reviewing the article and providing helpful feedback. Special thanks to the Human Computation editorial team for encouragement and support in the preparation of this article and this issue.

The CCL Summit was funded by the EU project Citizen Cyberlab (Grant No 317705).

\section{REFERENCES}

Abu Amsha, O., Schneider, D. K., Fernandez-Marquez, J. L., Da Costa, J., Fuchs, B., \& Kloetzer, L. (2016). Data Analytics in Citizen Cyberscience: Evaluating Participant Learning and Engagement with Analytics. Human Computation, 3(1).

Batey, M. (2012). The measurement of creativity: From definitional consensus to the introduction of a new heuristic framework. Creativity Research Journal. Retrieved from http://www.tandfonline.com/doi/abs/10.1080/10400419.2012.649181

Benony, M., Cardon, M., Ferré, A., Coquet, J., Foulquier, N., Thonier, F., ... Wintermute, E. H. (2016). The smell of us - crowdsourcing human body odor evaluation. Human Computation, 3(1).

Bonney, R., Cooper, C. B., Dickinson, J., Kelling, S., Phillips, T., Rosenberg, K. V., \& Shirk, J. (2009). Citizen Science: A Developing Tool for Expanding Science Knowledge and Scientific Literacy. BioScience, 59(11), 977-984. https://doi.org/10.1525/bio.2009.59.11.9

Brossard, D., Lewenstein, B., \& Bonney, R. (2005). Scientific knowledge and attitude change: The impact of a citizen science project. International Journal of Science Education, 27(9), 1099-1121. https://doi.org/10.1080/09500690500069483 
Cardamone, C., Schawinski, K., Sarzi, M., Bamford, S. P., Bennert, N., Urry, C. M., ... VandenBerg, J. (2009). Galaxy Zoo Green Peas: discovery of a class of compact extremely star-forming galaxies. Monthly Notices of the Royal Astronomical Society, 399(3), 11911205. https://doi.org/10.1111/j.1365-2966.2009.15383.x

Causer, T., \& Terras, M. (2014). Crowdsourcing Bentham: Beyond the Traditional Boundaries of Academic History. International Journal of Humanities and Arts Computing, 8(1), 46-64. https://doi.org/10.3366/ijhac.2014.0119

Crall, A. W., Jordan, R., Holfelder, K., Newman, G. J., Graham, J., \& Waller, D. M. (2013). The impacts of an invasive species citizen science training program on participant attitudes, behavior, and science literacy. Public Understanding of Science (Bristol, England), 22(6), 745-64. https://doi.org/10.1177/0963662511434894

Cronje, R., Rohlinger, S., Crall, A., \& Newman, G. (2011). Does Participation in Citizen Science Improve Scientific Literacy? A Study to Compare Assessment Methods. Applied Environmental Education \& Communication, 10(3), 135-145. https://doi.org/10.1080/1533015X.2011.603611

Curtis, V. (2015). Motivation to Participate in an Online Citizen Science Game: A Study of Foldit. Science Communication, 1075547015609322-. https://doi.org/10.1177/1075547015609322

Dickinson, H. (2011). Citizen Science: Science Needs YOU! The Naked Scientists. Retrieved January 22, 2016, from http:/www.thenakedscientists.com/HTML/articles/article/citizenscience-science-needs-you/

Fernandez-Marquez, J. L., Charalampidis, I., Abu-Amsha, O., Grey, F., Schneider, D. K., Segal, B., \& Mohanty, S. P. (2016). CCLTracker Framework: Monitoring user learning and activity in web based citizen science projects. Human Computation, 3(1).

Grey, F. (2009). Viewpoint: The age of citizen cyberscience. Cern Courier. Retrieved from https://scholar.google.ch/scholar?hl=en\&q=Viewpoint $\% 3 \mathrm{~A}+\mathrm{The}+$ age + of + citizen + cyberscie nce $\& b t n G=\&$ as_sdt $=1 \% 2 C 5 \&$ as_sdtp $=\# 0$

Haklay, M. (2013). Citizen Science and Volunteered Geographic Information - overview and typology of participation. (D. Sui, S. Elwood, \& M. Goodchild, Eds.). Dordrecht: Springer Netherlands. https://doi.org/10.1007/978-94-007-4587-2

Hanappe, P., Dunlop, R., Maes, A., Steels, L., \& Duval, N. (2016). Agroecology: A Fertile Field for Human Computation. Human Computation, 3(1).

Himmelstein, J., Goujet, R., Duong, T. K., Bland, J., \& Lindner, A. B. (2016). Improving Citizen Science Games through Open Analytics Data. Human Computation, 3(1).

Iacovides, I., Jennett, C., Cornish-Trestrail, C., \& Cox, A. L. (2013). Do games attract or sustain engagement in citizen science? In CHI'13 Extended Abstracts on Human Factors in Computing Systems on - CHI EA '13 (p. 1101). New York, New York, USA: ACM Press. 
https://doi.org/10.1145/2468356.2468553

Jennett, C., \& Cox, A. L. (2014, October 14). Eight Guidelines for Designing Virtual Citizen Science Projects. Second AAAI Conference on Human Computation and Crowdsourcing. Retrieved from http://www.aaai.org/ocs/index.php/HCOMP/HCOMP14/paper/view/9261

Jennett, C., Eveleigh, A., Mathieu, K., Ajani, Z., \& Cox, A. (2013, June 5). Creativity in citizen science: All for one and one for all. Retrieved from http://discovery.ucl.ac.uk/1395531/

Jennett, C., Kloetzer, L., Cox, A. L., Schneider, D. K., Collins, E., Fritz, M., ... Charalampidis, I. (2016). Creativity in Online Citizen Science: Interviews with Volunteers of the Citizen Cyberlab Pilot Projects. Human Computation, 3(1).

Jennett, C., Kloetzer, L., Schneider, D., Iacovides, I., Cox, A., Gold, M., ... Talsi, Y. (2016). Motivations, learning and creativity in online citizen science. Journal of Science Communication, 15 (3) , Article A05. (2016) .

Johnson, N. E. (2016). Landfill Hunter: Learning about Waste through Public Participation. Human Computation, 3(1).

Jordan, R. C., Gray, S. A., Howe, D. V, Brooks, W. R., \& Ehrenfeld, J. G. (2011). Knowledge gain and behavioral change in citizen-science programs. Conservation Biology: The Journal of the Society for Conservation Biology, 25(6), 1148-54. https://doi.org/10.1111/j.1523-1739.2011.01745.x

Khatib, F., DiMaio, F., Cooper, S., Kazmierczyk, M., Gilski, M., Krzywda, S., ... Baker, D. (2011). Crystal structure of a monomeric retroviral protease solved by protein folding game players. Nature Structural \& Molecular Biology, 18(10), 1175-1177. https://doi.org/10.1038/nsmb.2119

Kloetzer, L., Da Costa, J., \& Schneider, D. K. (2016). Not so passive: engagement and learning in Volunteer Computing projects. Human Computation, 3(1).

Kloetzer, L., Schneider, D., Jennett, C., Iacovides, I., Eveleigh, A., Cox, A., \& Gold, M. (2013, February 3). Learning by volunteer computing, thinking and gaming: What and how are volunteers learning by participating in Virtual Citizen Science? ESREA 2013: Changing Configurations of Adult Education in Transitional Times Proceedings. Retrieved from http://discovery.ucl.ac.uk/1418570/

Lave, J., \& Wenger, E. (1991). Situated Learning: Legitimate Peripheral Participation. Cambridge University Press. Retrieved from https://books.google.com/books?id=CAVIOrW3vYAC\&pgis=1

Lopez Martinez, D., Lombraña, D., Grey, F., \& Hwu, E. (2016). A Crowdsourcing-based Air Pollution Measurement System Using DIY Atomic Force Microscopes. Human Computation, 3(1).

Marsick, V. J., \& Watkins, K. E. (2001). Informal and Incidental Learning. New Directions for Adult and Continuing Education, 2001(89), 25. https://doi.org/10.1002/ace.5 
Philip Bell, Bruce Lewenstein, Andrew W. Shouse, M. A. F. (2009). Learning science in informal environments : people, places, and pursuits - Committee on Learning Science in Informal, Environments. https://doi.org/10.17226/12190

Price, C. A., \& Lee, H.-S. (2013). Changes in participants' scientific attitudes and epistemological beliefs during an astronomical citizen science project. Journal of Research in Science Teaching, 50(7), 773-801. https://doi.org/10.1002/tea.21090

Roick, O., Haklay, M., \& Ellul, C. D. (2016). GeoKey - open infrastructure for community mapping and science. Human Computation, 3(1).

Rotman, D., Preece, J., Hammock, J., Procita, K., Hansen, D., Parr, C., ... Jacobs, D. (2012). Dynamic Changes in Motivation in Collaborative Citizen-Science Projects. In Proceedings of the ACM 2012 conference on Computer Supported Cooperative Work - CSCW'12 (pp. 217-226). New York, USA: ACM Press. https://doi.org/10.1145/2145204.2145238

Silvertown, J. (2009). A new dawn for citizen science. Trends in Ecology \& Evolution (Personal Edition), 24(9), 467-71. https://doi.org/10.1016/j.tree.2009.03.017

Vygotsky, L. S., \& Cole, M. (1978). Mind in Society. Harvard University Press. Retrieved from https://books.google.com/books?id=RxjjUefze_oC\&pgis=1

Yadav, P., \& Darlington, J. (2016a). Conceptual Frameworks for Building Online Citizen Science Projects. Human Computation, 3(1).

Yadav, P., \& Darlington, J. (2016b). Design Guidelines for the User-Centred Collaborative Citizen Science Platforms. Human Computation, 3(1). 